\title{
FORECASTING INNOVATION DIFFUSION WITH NEAR-OPTIMAL BERTALANFFY-PÜTTER MODELS
}

\author{
Manfred Kühleitner ${ }^{*}{ }^{\natural}$, Norbert Brunner ${ }^{2}$, Katharina Renner-Martin ${ }^{2}$ \\ ${ }^{*} 1,2$ Department of Integrative Biology and Biodiversity Research (DIBB), University of Natural \\ Resources and Life Sciences (BOKU), Austria
}

DOI: https://doi.org/10.29121/ijetmr.v7.i8.2020.745

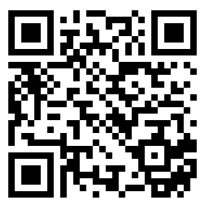

Article Citation: Manfred

Kühleitner, Norbert Brunner, and

Katharina Renner-Martin. (2020).

FORECASTING INNOVATION

DIFFUSION WITH NEAR-OPTIMAL

BERTALANFFY-PÜTTER MODELS.

International Journal of Engineering

Technologies and Management

Research, 7(8), 1-11.

https://doi.org/10.29121/ijetmr.v7

i8.2020.745

Published Date: 07 August 2020

Keywords:

Akaike Weight

Bertalanffy-Pütter Differential

Equation

Least Squares

Near-Optimal Models

Forecasting

Model-Uncertainty

\section{Abbreviations:}

Aic- Akaike Information Criterion

Bp-Model- Bertalanffy-Pütter Model

Sse- Sum of Squared

Errors

\begin{abstract}
Using a classical example for technology diffusion, the mechanization of agriculture in Spain since 1951, we considered the forecasting-intervals from the near-optimal Bertalanffy-Pütter (BP) models. We used BPmodels, as they considerably reduced the hitherto best fit (sum of squared errors) reported in literature. And we considered near-optimal models (their sum of squared errors is almost best), as they allowed to quantify model-uncertainty. This approach supplemented traditional sensitivity analyses (variation of model parameters), as for the present models and data even slight changes in the best-fit parameters resulted in very poorly fitting model curves.
\end{abstract}

\section{INTRODUCTION}

\subsection{BACKGROUND}

Technology forecasting uses a wide range of methods (Firat et al., 2008). This paper focuses on a popular phenomenological approach, trend analysis for the diffusion of technologies by means of sigmoidal (S-shaped) model curves. Here, an often considered three-parameter model was the Verhulst model of logistic growth (e.g. Adamuthe \& Thampi, 2019; Naseri \& Elliott, 2013; Yamakawa et al., 2013). This paper proposes a five-parameter model to describe the success (growth, diffusion) of a technology, the Bertalanffy-Pütter (BP) model. It generalizes many

(C) 2020 The Author(s). This is an open access article distributed under the terms of the Creative Commons Attribution License, which permits unrestricted use, distribution, and reproduction in any medium, provided the original author and source are credited. 
conventional three-parameter models (e.g. logistic growth) and therefore improves their fit. In addition, the two additional parameters allow to identify near-optimal three-parameter models that do not differ significantly from the best-fit BP-model. Comparing the forecasts from these near-optimal models with the forecast from the best-fit model results in new quantitative indicators for assessing model-uncertainty ex ante. We illustrate this approach for data from literature, where we explore, which levels of model-uncertainty occurred in the past for forecasting. We expect that such empirical studies will lead to sensible recommendations for practitioners about what modeluncertainty needs to be assumed for different timespans of prognosis.

\subsection{PROBLEM}

Model uncertainties arise, when gaps in knowledge about the true drivers and mechanisms of growth cannot be reduced by an analysis of the past observations. For instance, as was observed for the prognosis of cancer, many growth curves with different shapes in the future may fit well to given historic data (Kühleitner et al., 2019). The paper develops a new approach to assess model-uncertainty and it illustrates it for forecasting. In order to measure model-uncertainty quantitatively, Bai \& Jin (2016) suggested to use the relative error of the prediction. However, ex ante we could not compute this future error of prediction.

We propose a different approach. We identify models that fit well to the current data and that therefore in a technical sense defined below have a certain "probability to be true". Thereby, we use the Akaike information criterion AIC (Akaike, 1974) and its associated probability (Akaike weights): For given data, the model with the lowest $A I C$ is most likely to be true and comparing other models with this one, their probabilities to be true are computed from the differences in AIC.

Based on this notion, we define a forecasting interval by the lower and upper bounds of the predictions drawn from likely models. Thereby, we consider all BP-models with a certain probability to be true, when compared to the best-fitting one. The forecasts based on these models define the forecasting interval. This concept resembles the confidence interval, but the confidence interval assumes a fixed model that is fitted to random variations of the data, while for the forecasting interval the data remain fixed.

\section{MATERIALS AND METHODS}

\subsection{MATERIALS}

The computations used Mathematica 12.0 software of Wolfram Research. The results of optimization were exported to a spreadsheet (Microsoft Excel) and reimported into Mathematica for further analysis of the modeluncertainty.

\subsection{DATA}

We use data about the mechanization of agriculture in Spain by means of tractor ownership. The primary sources were census data and government reports over the period 1951-1976 (Mar-Molinero, 1980). While the data may appear to be outdated, they cover an interesting phase for agriculture in Spain: During the early 1950s, the ancient regime of Franco gave up its disastrous policy of economic autarky and in the 1960s and 70s this was followed by a policy of modernization of the agricultural sector (Táboas et al., 2019). Further, the data for 1951-1976 were used repeatedly to test approaches to forecasting (e.g. Nguimkeu, 2014; Franses, 1994; Meade, 1984; MarMolinero, 1980). Gurung et al. (2018) is a recent related study about the mechanization of agriculture in India.

The data were rescaled to start at $t=0$, meaning the year 1951. (This rescaling was used in literature.) We used equation (1) to model the stock of tractors $y(t)$ over time $t$ in years. Thereby, we first searched for the best fitting BP-model for the data till 1976. In order to assess prognosis, we also identified the best fitting BP-models to ten truncated data (number of tractors till the year 1966, 1967, ... 1975) that we used to test the forecast for 1976 in hindsight. We supplemented this example by considering in addition the 1977-2009 data from the World Bank Open Data repository starting in 1961 (World Bank, 2019) and identifying the best-fit BP model for the extended data till 2009. The combined data are listed in Table 1. 
Manfred Kühleitner, Norbert Brunner, and Katharina Renner-Martin

Table 1: Stock of tractors in Spain during the period 1951-2009. ${ }^{1}$

\begin{tabular}{|c|c|c|c|c|c|c|c|c|c|c|c|}
\hline Year $^{2}$ & Stock $^{3}$ & Year & Stock & Year & Stock & Year & Stock & Year & Stock & Year & Stock \\
\hline 0 & 1.3800 & 10 & 7.1080 & 20 & 28.2370 & 30 & 54.8080 & 40 & 75.5740 & 50 & 92.5690 \\
\hline 1 & 1.5800 & 11 & 9.2750 & 21 & 30.6190 & 31 & 57.1170 & 41 & 76.6270 & 51 & 94.6050 \\
\hline 2 & 1.9000 & 12 & 11.4410 & 22 & 33.0360 & 32 & 59.2010 & 42 & 77.5300 & 52 & 94.3650 \\
\hline 3 & 2.1440 & 13 & 13.0130 & 23 & 35.5550 & 33 & 61.1430 & 43 & 78.9750 & 53 & 96.6600 \\
\hline 4 & 2.6020 & 14 & 14.7880 & 24 & 37.9070 & 34 & 63.3210 & 44 & 80.5590 & 54 & 98.0810 \\
\hline 5 & 2.9960 & 15 & 16.9190 & 25 & 40.0928 & 35 & 65.7830 & 45 & 82.3610 & 55 & 100.0220 \\
\hline 6 & 3.4180 & 16 & 18.0720 & 26 & 42.1390 & 36 & 67.8680 & 46 & 84.1930 & 56 & 101.6040 \\
\hline 7 & 4.0680 & 17 & 20.4360 & 27 & 45.5680 & 37 & 70.1930 & 47 & 86.2140 & 57 & 103.0440 \\
\hline 8 & 4.7080 & 18 & 23.4540 & 28 & 49.1600 & 38 & 72.2660 & 48 & 88.1980 & 58 & 103.8730 \\
\hline 9 & 5.6840 & 19 & 25.9820 & 29 & 52.3910 & 39 & 74.0830 & 49 & 89.9700 & & \\
\hline
\end{tabular}

1) Source: data combined from Figure 5 of Mar-Molinero (1980) and open data from World Bank (2019).

2) Start with 1951 (year 0); 1976 corresponds to year 25.

3) One unit is a stock of 10,000 tractors.

\subsection{BERTALANFFY-PÜTTER (BP) MODEL}

The growth function $y(t)$ of the BP-model is a solution of the following differential equation (Pütter, 1920), which can be solved analytically, though in general not by means of elementary functions (Ohnishi et al., 2014).

$$
y^{\prime}(t)=p \cdot y(t)^{a}-q \cdot y(t)^{b}
$$

The five model parameters are determined from fitting the model to historical data: Four parameters are displayed in the equations, namely the non-negative exponent-pair $a<b$ and the constants $p$ and $q$. An additional parameter is the initial value, i.e. $y(0)=c>0$.

Each exponent-pair $(a, b)$ defines a unique BP-model $B P(a, b)$ with three free parameters. Well-known examples are bounded exponential growth $B P(0,1)$, logistic growth $B P(1,2)$, the von Bertalanffy model $B P(1,2 / 3)$, and the West model $B P(1,3 / 4)$. The Solow (1957) model of economic growth is a class of BP-models; it coincides with the generalized Bertalanffy model $(b=1$ and $a<1)$. Also, the Richards model is a class of BP-models $(a=1$ and $b>1)$. The Gompertz-model is the limit case $B P(1,1)$ with a different differential equation, where $b$ converges to $a=$ 1 from above (Marusic \& Bajzer, 1993). These models have been used in forecasting, e.g. business trends (Dhakal, 2018), tumor growth (Murphy at al., 2016), or epidemic trajectories (Pell et al., 2018). In biology, these models have been proposed to model the growth of plants (Richards, 1959) or the mass growth of vertebrates (e.g. West et al., 2001; Bertalanffy, 1949).

In literature, there are several other five-parameter growth models, such as the model of Bass (1969) for market diffusion or the model of Monod (1949) for bacterial kinetics. We decided to use the BP-model, as it was very flexible. In comparison to other five-parameter models, this versatility had the disadvantage that for the BP-model the standard optimization tools (e.g. command Non-Linear Model Fit in Mathematica) did not always identify the bestfit parameters (numerical instability). However, as explained below, we could overcome this difficulty, whence this model is now feasible for practitioners. As to another limitation, the BP-model is not suitable in situations, where both growth and decay occur. Rather, it is intended to improve the fit to the data in situations, where initially e.g. logistic growth has been considered as a viable model.

\subsection{DATA FITTING}

The most common method for data-fitting, used also for this paper, is the method of least squares, which fits a (nonlinear) model to past data (Satoh \& Matsumura, 2018). Thus, model selection aimed at finding parameters that minimized the sum of squared errors $S S E$. If $y(t)$ is a solution of equation (1), using certain exponents $a<b$ and parameters $p, q, c$, and if $\left(t_{i}, y_{i}\right)$ are the $N$ data, then $S S E$ is defined by equation (2): 
Forecasting Innovation Diffusion with Near-Optimal Bertalanffy-Pütter Models

$$
S S E=\sum_{i=1}^{N}\left(y_{i}-y(t)\right)^{2}
$$

As explained above, for model (1) the standard optimization tools did not find best-fit parameters to minimize (2). We did overcome this difficulty by considering exponent-pairs $(a, b)$ on a grid with step size 0.01 in both directions; initially $0 \leq a \leq 2.5, a<b \leq a+3.5$. (The grid was adaptively enlarged during optimization; for one dataset we searched about 200,000 grid points.) Figure 1 plots the search-grid (yellow area) and it displays the exponent-pairs of named models by dots (e.g. Verhulst model) or lines (e.g. Richards' model interpreted as a model class). Compared to the search-grid, these models were rather exceptional.

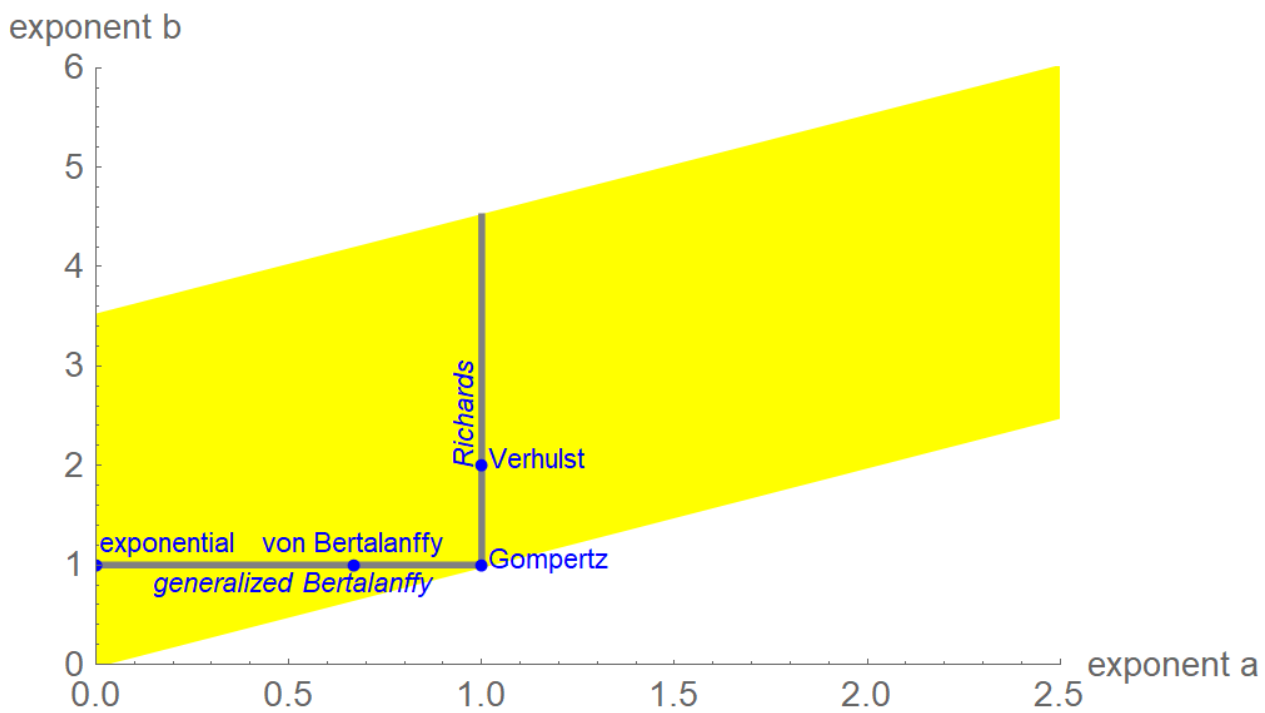

Figure 1: Named models (blue) and model classes (italics and lines) and part of the search region (yellow) of BP-models (plot using Mathematic 12.0).

For each exponent-pair of the grid we identified the best fitting model parameters $(p, q, c)$ that minimized SSE. This defined the following function of equation (3), assuming for the minimization (right hand side) model (1) with exponents $a, b$ :

$$
S S E_{o p t}(a, b)=\min _{c, p, q}(S S E)
$$

The best fitting model had the overall least sum of squared errors ( $S S E_{\min }$ ). We identified its exponent-pair $\left(a_{\min }, b_{\min }\right)$ with an accuracy of 0.01 (as we searched only grid-points) and its parameters $p_{\min }, q_{\min }, c_{\min }$ that minimized $S S E_{\text {opt }}\left(a_{\min }, b_{\min }\right)=S S E_{\min }$.

For each grid-point $(a, b)$, the optimization of $p, q$, and $c$ was done using a custom-made variant of the method of simulated annealing (Vidal, 1993). The details and the code were outlined in another paper (Renner-Martin et al., 2018, Kühleitner et al., 2019). The outcome was exported into a spreadsheet, whose rows listed the best fit parameters $a, b, c, p, q$, and $S S E$ for each grid-point. The exponent-pair $\left(a_{\min }, b_{\min }\right)$ was identified from the row, where the least value of $S S E$ was attained.

\subsection{COMPARISON OF MODELS}

We use SSE of equation (2) as the primary measure to assess the fit of a model to data. Related measures used in forecasting literature (for a survey: Hyndman \& Koehler, 2006) are the root-mean-square error, $R M S E=\sqrt{\frac{S S E}{N}}$, and R-squared, $R^{2}=1-\frac{S S E}{S Q T}$. Here, $S Q T$ is the total sum of squares (variance of the $N$ data $y_{i}$ times $N-1$ or $N$, depending on the estimator for the variance). The use of SSE was based on assuming normally distributed errors. 
Manfred Kühleitner, Norbert Brunner, and Katharina Renner-Martin

Different statistical assumptions have led to weighted sums of squared errors. In addition, we used the Akaike information criterion, $A I C=N \cdot \ln (S S E / N)+2 \cdot K$; c.f. Burnham \& Anderson (2002) or Motulsky \& Christopoulos (2003). Here, $N$ is the number of the data-points. For the 1951-1976 data, $N=26$; for the truncated data, $N$ ranges from 16 to 25; and for the 1951-2009 data, $N=59$. Further, $K=4$ is the number of optimized parameters $(c, p, q$ and $S S E$ as an implicit parameter). As we compared a finite set of three-parameter BP-models $B P(a, b)$ using exponentpairs chosen from a grid, we did not penalize the best fitting model by a higher number of parameters (the optimal exponent-pair was roughly approximated, but not yet computed). Thus, for these models, the least $A I C_{\min }$ was achieved for the model defined from the exponent-pair $\left(a_{\min }, b_{\min }\right)$.

If the model with $A I C_{\min }$ is compared to another model with larger $A I C$, then the probability that the other model is true (in an information theoretic meaning) is given by the relative Akaike weight $e^{-d / 2} /\left(1+e^{-d / 2}\right)$, using the difference $d=A I C-A I C_{\min }$. This probability is at most $50 \%$ (comparing the model with the least $A I C$ with an almost as good model).

\subsection{FORECASTING INTERVALS}

The starting point of our new approach are the data and $S S E_{\min }$ together with all $u$-near-optimal exponent-pairs $(a, b)$. Thereby, an exponent-pair $(a, b)$ is $u$-near-optimal with model-uncertainty $u$, if $\operatorname{SSE}_{\text {opt }}(a, b) \leq(1+u) \cdot S S E_{\text {min }}$. For each exponent-pair we also consider the best fitting growth curve $y_{a, b}(t)$; it is specified by its parameters $a, b, c, p, q$. All models that are displayed in the yellow search region of Figure 1 by their exponent-pairs are meant to realize the best possible fit to the given data, i.e. depending on $a$ and $b$ (which defined the model) the model parameters $p, q$ and $c$ were optimized according formula (3). Therefore, for the data that represent the past, there was barely a difference between the model curves for models whose $S S E$ did not differ much from $S S E_{\text {min }}$.

In analogy to confidence intervals we now define: For a point of time $T$ the forecasting interval $I_{u}(T)$ for the level $u$ of model-uncertainty has as its end-points the least upper and the largest lower bounds of the function values $y_{a, b}(T)$ associated to $u$-near-optimal exponent-pairs. For the computation of a forecasting interval $I_{u}(t)$, filtering in the table of optimal parameters identified the rows with $S S E \leq(1+u) \cdot S S E_{\min }$. The parameters of each of these rows defined a growth function $y_{a, b}$ that was evaluated at $T$. The minimum and maximum of these values defined the boundaries of the interval.

In order to obtain a closer analogy to the confidence intervals, we related model-uncertainty in the following way to the probability that a model is true in the information theoretic sense, using $A I C$ and more specifically, the relative Akaike weight. For, as follows from the above section, if the exponent-pair $(a, b)$ is near-optimal with modeluncertainty $u$, then the probability that this exponent-pair is true is given by the following formula (4) for the relative Akaike-weight.

$$
\operatorname{prob}(a, b)=\frac{1}{1+\sqrt{(1+u)^{N}}}
$$

\section{RESULTS AND DISCUSSIONS}

\subsection{PREVIOUS OUTCOMES}

The trend for tractors has been studied repeatedly. There is a consensus in literature that the growth of tractors would follow a logistic model. Mar-Molinero (1980) compared several models and for the 1951-1976 tractor data he reported $S S E=4.9768$ as the best fit, obtained by the logistic model. Subsequent authors (e.g. Meade, 1984; Franses, 1994; Nguimkeu, 2014) confirmed this conclusion.

Mar-Molinero (1980) also reported an "unexplained residual sum of squares" of 2.57. However, he referred to autocorrelation, using a fit of a time series: $r_{t}=\rho \cdot r_{t-1}+\varepsilon_{t}$ for the residuals $r_{t}=y_{t}-y(t)$ and the unexplained error $\varepsilon_{t}$. This paper aims at improving the fit by using a better growth curve, but it does not follow up on the autocorrelation. 


\subsection{DATA FITTING}

In order to identify the best-fit BP-model for the 1951-1976 tractor data, and for the truncated data, we searched between $0.9-1.3 \times 10^{5}$ grid-points. Figure 2 plots the best fitting growth curves. Their best-fit parameters are listed in Table 2 and their exponent-pairs are plotted in Figure 3.

Table 2: Parameters for the best fit of model (1) to the data from 1951 up to the indicated year.

\begin{tabular}{|c|c|c|c|c|c|c|c|c|}
\hline \multirow{2}{*}{ Year $^{1}$} & \multicolumn{5}{|c|}{ Best fit parameters } & \multicolumn{3}{|c|}{ Goodness of fit } \\
\cline { 2 - 9 } & $a_{\min }$ & $b_{\min }$ & $c_{\min }$ & $p_{\min }$ & $q_{\min }$ & $S S E_{\min }$ & $R M S E$ & $R^{2}$ \\
\hline 1966 & 1.91 & 2.12 & 1.47507 & 0.182778 & 0.0976106 & 0.581875 & 0.190702 & 0.998599 \\
\hline 1967 & 1.89 & 2.13 & 1.47848 & 0.166224 & 0.0808923 & 0.58954 & 0.186223 & 0.998926 \\
\hline 1968 & 1.72 & 1.9 & 1.37506 & 0.252761 & 0.14337 & 1.29791 & 0.268526 & 0.998208 \\
\hline 1969 & 1.42 & 1.62 & 1.20355 & 0.312459 & 0.155705 & 2.92583 & 0.392417 & 0.996969 \\
\hline 1970 & 1.34 & 1.42 & 1.13237 & 0.700413 & 0.519034 & 3.53807 & 0.420599 & 0.997196 \\
\hline 1971 & 1.24 & 1.4 & 1.08056 & 0.423065 & 0.227006 & 3.64734 & 0.416753 & 0.997732 \\
\hline 1972 & 1.22 & 1.38 & 1.08142 & 0.424588 & 0.22552 & 3.69308 & 0.409716 & 0.998164 \\
\hline 1973 & 1.17 & 1.4 & 1.06337 & 0.341165 & 0.136074 & 3.74722 & 0.403637 & 0.998487 \\
\hline 1974 & 1.17 & 1.37 & 1.07238 & 0.37085 & 0.165032 & 3.84279 & 0.400145 & 0.998725 \\
\hline 1975 & 1.15 & 1.34 & 1.03507 & 0.396099 & 0.182001 & 3.89954 & 0.394945 & 0.998921 \\
\hline 1976 & 1.12 & 1.32 & 0.997355 & 0.396746 & 0.173354 & 3.91475 & 0.38803 & 0.999085 \\
\hline 2009 & 0.75 & 1.02 & 0.253028 & 0.590051 & 0.159631 & 64.9233 & 1.049 & 0.999085 \\
\hline
\end{tabular}

1) This indicates the data from 1951 to the displayed year

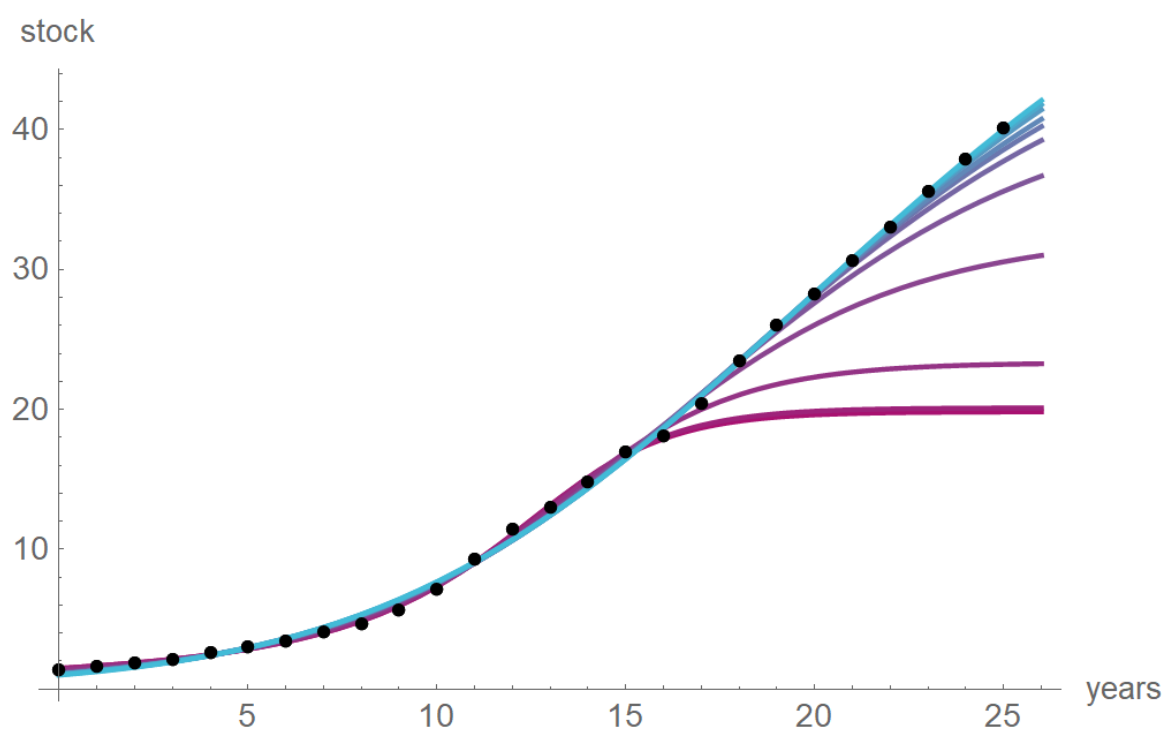

Figure 2: Stock of tractors for 1951-1976 (black dots) with the best fitting growth curve of the BP-class (top line) and forecasts using the best fitting growth curves for the data till 1966, 1967, ... 1975 (increasing order of the curves); the best fit parameters are from Table 2 (plot using Mathematica 12.0).

Judging from $R^{2}$ (Table 2), for all (truncated) data the fit of all curves to these data was excellent (99.7-99.9\% of the variance in the data was explained by the models). Further, the best fitting model curve for the 1951-1976 data with SSE $=3.91475$ displayed a significant improvement of $21 \%$ over the previous $S S E=4.9768$ reported in literature for logistic growth. However, the extrapolations of the growth curves for the truncated data to the remaining data underestimated the future growth (Figure 2). In particular, the best fitting growth curves to the data till 1966, 1967, ... and 1969 did rapidly approach their asymptotic limits and therefore their prognosis for $T=1976$ remained considerably below the true value. However, the error became smaller, the more data were used. Further, the best fit exponent-pairs for different years were spread widely along a regression line (Figure 3). For comparison, we also plotted the optimal exponents for the extended data 1951-2009. 


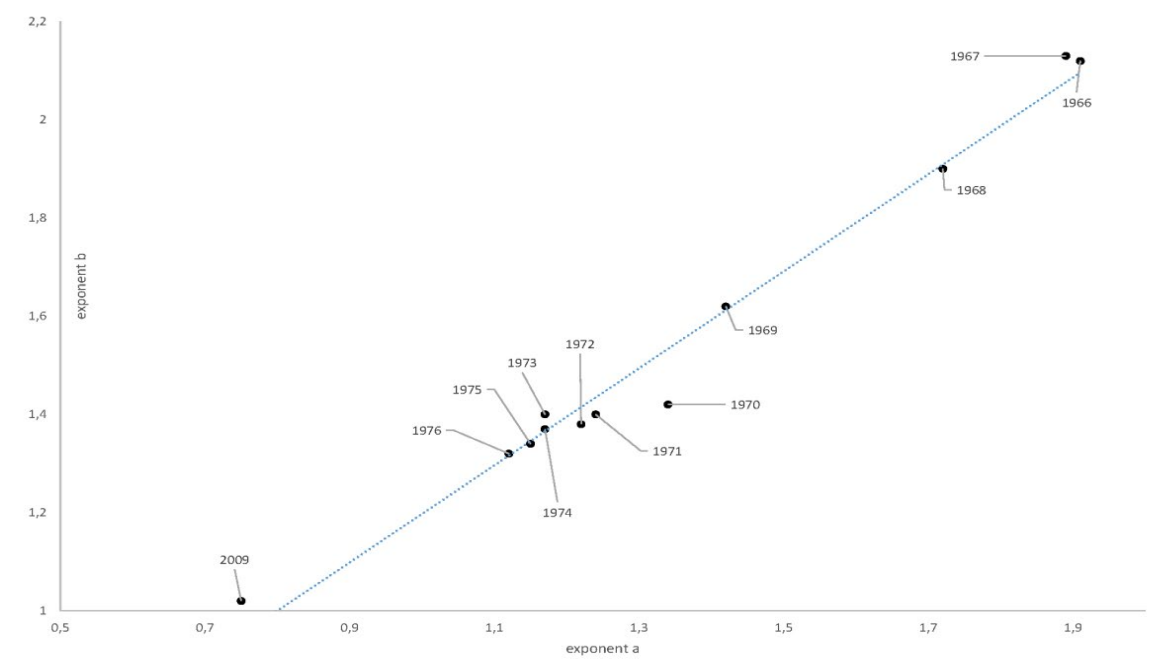

Figure 3: Optimal exponent-pairs (Table 2) and linear trend $b=0.2094+0.988 \cdot a$ (dotted, plot using MS Excel).

\subsection{FORECASTING}

Figure 4 displays the near-optimal exponent-pairs for the truncated data for 1951-1971. This figure was obtained as a by-product of our approach to data-fitting, where for each of almost 127,000 exponent-pairs $(a, b)$ the best-fit exponents to the truncated data were found by an optimization. The red area displays about 2700 nearoptimal exponent-pairs, for which $S S E_{\text {opt }}$ did not exceed the overall best $S S E_{\min }$ (attained at the black dot) by more than $u=10 \%$. The blue area corresponds to the near-optimal exponent-pairs with $u=34 \%$. The best fitting models using these exponent-pairs have a probability of $5 \%$ or more to be true, when compared to the best-fit model. We repeated these computations for the truncated tractor data till 1970,1971, .. and 1975. (In view of Figure 2, the data truncated at 1969 or earlier were unsuitable for prognosis and in view of Figure 3 their best-fit exponent-pairs were remote from the exponent-pair for the data till 1976.)

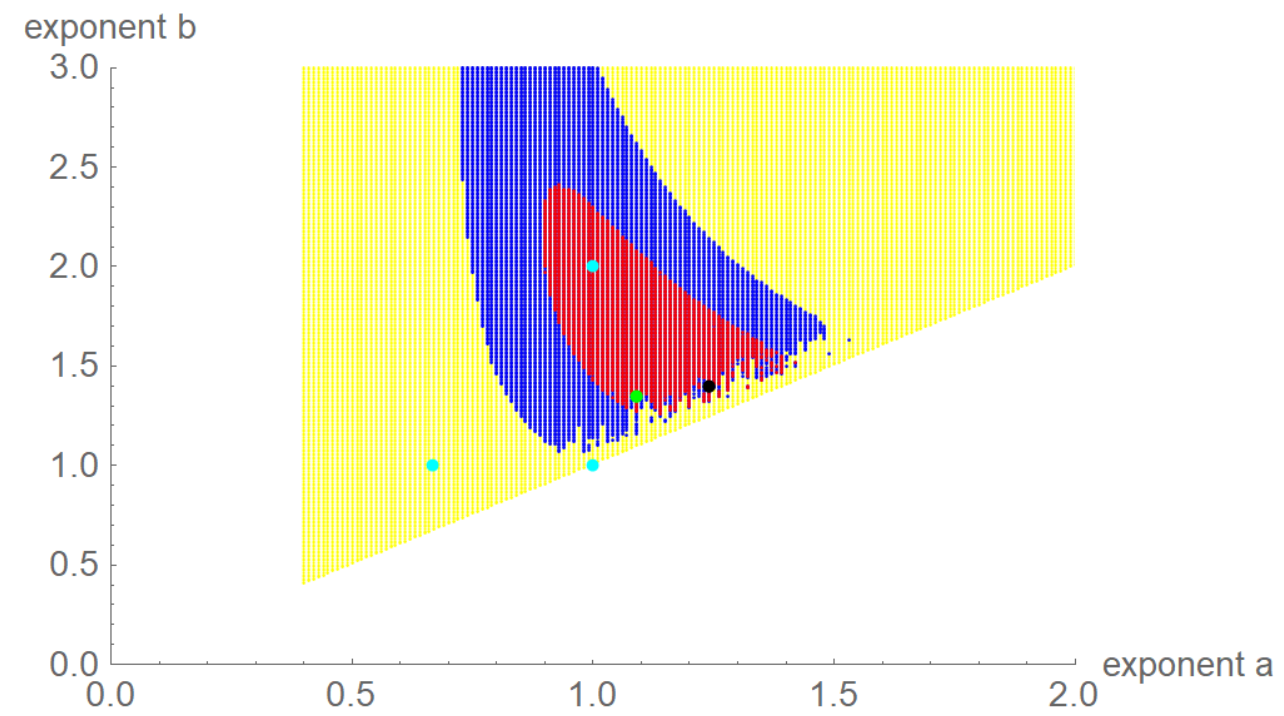

Figure 4: Part of the searched grid points (yellow) for the fit of model (1) to the tractor data for 1951-1971, near-optimal exponent-pairs assuming a model-uncertainty of $u=34 \%$ (blue: this corresponds to a probability of $5 \%$ ) and of $u=10 \%$ (red: probability $28 \%$ ), optimal exponent-pair for these data (black, see Table 2), exponent- 
pair to obtain an upper bound for the prognosis for 1976 (green, see Table 3) and selected exponent-pairs of named models (light blue: logistic, Gompertz and von Bertalanffy); plot using Mathematica 12.0.

Next, for each of near-optimal exponent-pair $(a, b)$ the best fitting growth curve $y_{a, b}(t)$ was identified and its "future" values were also evaluated ("future" referring to the perspective of the fitted data). Their upper and lower bounds defined forecasting intervals. Figure 5 plots the resulting forecasting band corresponding to Figure 4, prognosis for the stock of tractors based on the data for 1951-1971. For the present data and the chosen modeluncertainty all data points from 1972-1976 (plotted in green) were within this forecasting band.

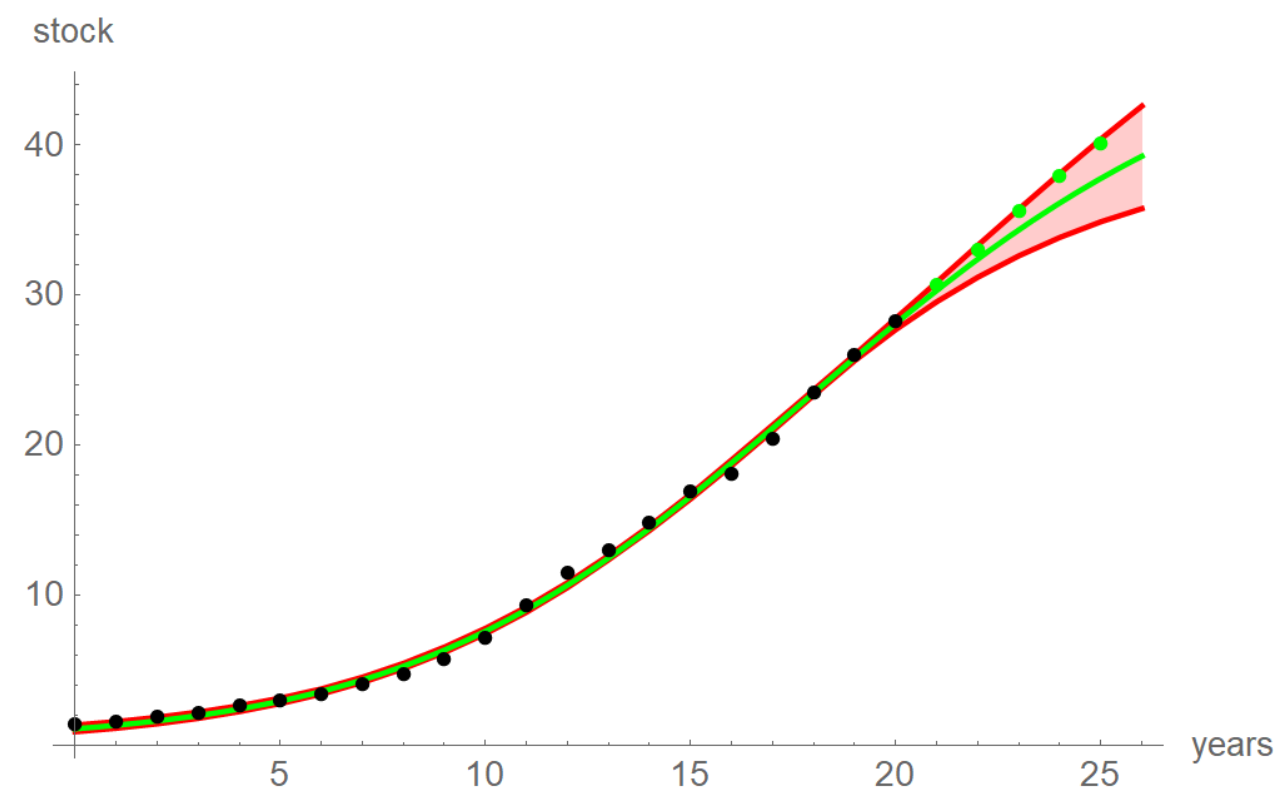

Figure 5: Forecasting band (red, defined from the red dots in Figure 4) around the best fitting growth curve (green) to the tractor data for 1951-1971 (black), and comparison with the data for 1972-1976 (green), assuming a model-uncertainty of $u=10 \%$; plot using Mathematica 12.0 .

These computations were repeated for all truncated data. Table 3 summarizes these results in the following way: It identifies the least model uncertainty that was needed to include the true value for 1976 in the forecasting interval. For instance, for each red exponent-pair $(a, b)$ of Figure 5 (model-uncertainty $u=10 \%$ ) the value of the growth curve $y_{a, b}$ was evaluated for the year 1976; the values ranged between 34.9 and 40.4 . The observed value at 1976 was 40.1 and the best-fitting curve to the 1951-1971 data attained a lower value. For Table 3 (line for 1971) we identified all model curves $y_{a, b}$ that attained a higher value and selected the one with the least SSE (Table 3 displays it as "needed $S S E^{\prime \prime}$ ).

Table 3: Minimal model-uncertainty so that the true value for 1976 was in the forecasting interval.

\begin{tabular}{|c|c|c|c|c|c|c|c|c|c|}
\hline \multirow[t]{2}{*}{ Year } & \multicolumn{5}{|c|}{ Model that achieves the required bound ${ }^{1}$} & \multirow{2}{*}{$\begin{array}{l}\text { Needed } \\
{ }^{2} S S E\end{array}$} & \multirow{2}{*}{$\begin{array}{c}\text { Logistic }^{3} S S E_{\text {logisti }} \\
c\end{array}$} & \multirow{2}{*}{$\begin{array}{c}\text { Model- } \\
\text { uncertainty } \\
4\end{array}$} & \multirow{2}{*}{$\begin{array}{c}\text { Probability } \\
4\end{array}$} \\
\hline & $a$ & $b$ & $c$ & $p$ & $q$ & & & & \\
\hline 1970 & $\begin{array}{c}1.1 \\
9 \\
\end{array}$ & $\begin{array}{c}1.2 \\
7 \\
\end{array}$ & 1.04613 & 0.76236 & 0.547886 & $\begin{array}{c}3.8450 \\
8 \\
\end{array}$ & 3.74498 & $8.677 \%$ & $30.3197 \%$ \\
\hline $\begin{array}{c}1971 \\
5 \\
\end{array}$ & $\begin{array}{c}1.0 \\
9 \\
\end{array}$ & $\begin{array}{c}1.3 \\
5\end{array}$ & $\begin{array}{c}0.98425 \\
8 \\
\end{array}$ & 0.34281 & 0.116463 & $\begin{array}{c}3.9023 \\
2 \\
\end{array}$ & 3.83045 & $6.991 \%$ & $32.9706 \%$ \\
\hline 1972 & 1.1 & $\begin{array}{c}1.3 \\
1 \\
\end{array}$ & $\begin{array}{c}0.98896 \\
6 \\
\end{array}$ & $\begin{array}{c}0.38888 \\
5 \\
\end{array}$ & 0.161407 & 3.959 & 3.92061 & $7.201 \%$ & $31.7592 \%$ \\
\hline 1973 & $\begin{array}{c}1.0 \\
8 \\
\end{array}$ & $\begin{array}{c}1.2 \\
9 \\
\end{array}$ & $\begin{array}{c}0.94000 \\
7 \\
\end{array}$ & $\begin{array}{c}0.40638 \\
2 \\
\end{array}$ & 0.168379 & $\begin{array}{c}3.9978 \\
5 \\
\end{array}$ & 4.05518 & $6.689 \%$ & $32.2009 \%$ \\
\hline 1974 & $\begin{array}{c}1.1 \\
2\end{array}$ & $\begin{array}{c}1.2 \\
6\end{array}$ & $\begin{array}{c}0.95506 \\
5\end{array}$ & $\begin{array}{c}0.52504 \\
2\end{array}$ & 0.29214 & $\begin{array}{c}3.9584 \\
6\end{array}$ & 4.35074 & $3.01 \%$ & $41.1955 \%$ \\
\hline
\end{tabular}




\begin{tabular}{|c|c|c|c|c|c|c|c|c|c|}
\hline 1975 & 1.0 & 1.3 & 0.99076 & 0.32237 & 0.096825 & 3.9483 & 4.68163 & $1.251 \%$ & $46.1232 \%$ \\
& 8 & 7 & 5 & 9 & 7 & 1 & & & \\
\hline
\end{tabular}

1) For each year, we searched for the exponent pair $(a, b)$, whose $\operatorname{SSE}_{\text {opt }}(a, b)$ was minimal subject to the constraint that its best fitting growth curve $y_{a, b}$ was above the 1976 value. The parameters of this $y_{a, b}$ are listed.

2) $S S E_{\text {opt }}$ of the displayed exponent-pairs; it was above $S S E_{\min }$ for the data (see Table 2) and "model uncertainty"

3) $\operatorname{SSE}_{\text {opt }}(1,2)$ of the logistic model.

4) The computations were based on $S S E_{\min }$ ( $7^{\text {th }}$ column of Table 2).

5) This model is represented by the green dot in Figure 4.

Table 3 shows that forecasting of the true value based on the truncated data did not require unlikely models: The probabilities of the used models ranged between $30-46 \%$ and $S S E_{\text {opt }}$ of these models exceeded $S S E_{\min }$ by $1-9 \%$. Thus, the prognosis for up to six years remained within the range of variability that could be expected from the data. Note that for the forecast based on the years till 1973, 1974, or 1975, the logistic model was outside this minimal range (i.e. $S S E_{\text {logistic }}$ exceeded the needed $S S E$ ).

\section{CONCLUSIONS AND RECOMMENDATIONS}

Forecasting requires the use of models that are capable to represent the hitherto observed data accurately. Model class (1) has some obvious advantages: it is a very flexible class of growth models and it includes a wide range of common growth models. Therefore, in general the best-fitting model of the BP-class will fit better than any of the above-mentioned named model. Thus, for the tractor data the use of models from the BP-class resulted in a significant improvement over the fit by the logistic growth function that was previously used in forecasting.

This approach requires extensive computations, where about hundred thousand models from the BP-class need to be optimized (different models are defined from different exponent-pairs). Yet, these optimization results serve an additional important purpose, as they may be used to quantify the model-uncertainty of forecasting. As is displayed by the forecasting intervals (Figure 5), the near-optimal model curves remained close to the data (on which data-fitting was based). Nevertheless, subsequently there were considerable differences.

For the present data it was feasible to consider forecasting intervals based on all models with a probability of $5 \%$ or more to be true. Using this approach, we have shown that the considered data were suitable for a prognosis over a time span of five years.

\section{SOURCES OF FUNDING}

None.

\section{CONFLICT OF INTEREST}

None.

\section{ACKNOWLEDGMENT}

The authors declare no competing interests. There occurred no ethical issues, as the research was based on published data. All authors contributed equally in research, evaluation and interpretation of the results and drafting the manuscript.

\section{APPENDICES}

The method section lists the data and explains their sources. Further, the authors provided the supplementary material, namely the following spreadsheet (MS Excel) with the outcomes of the optimizations. 
Forecasting Innovation Diffusion with Near-Optimal Bertalanffy-Pütter Models

S-File. Computation of SSEopt(a, b), based on Table 1 for the stock of tractors for the period 1951-1971, for certain grid-points, namely exponents $a$ and $b$, and for them the best fit-parameters (optimization results) initial number $\mathrm{m} 0, \mathrm{p}, \mathrm{q}$, and SSE.

\section{REFERENCES}

[1] Adamuthe, A.C., Thampi, G.K., 2019. Technology forecasting: A case study of computational technologies. Technological Forecasting \& Social Change 143, 181-189.

[2] Akaike, H., 1974. A New Look at the Statistical Model Identification. IEEE Transactions of Automatic Control 19, 716-723.

[3] Bai, Y., Jin, W.L., 2016. Marine Structural Design (2nd ed.) Elsevier, Amsterdam, Netherlands.

[4] Bass, F.M., 1969. A new product growth model for consumer durables. Management Science 15, 215-227.

[5] Bertalanffy, L.V., 1949. Problems of organic growth. Nature 163, 156-158.

[6] Burnham, K.P.; Anderson, D.R., 2002. Model Selection and Multi-Model Inference: A Practical InformationTheoretic Approach. Springer, Berlin.

[7] Dhakal, T., 2018. An analytical model on business diffusion. Journal of Industrial Engineering and Management Science 2018, 119-128. DOI 10.13052/jiems2446-1822.2018.007.

[8] Firat, A.K., Madnick, S., Woon, W.L., 2008. Technology forecasting: A review. In: Working Paper CISL\# 200815. MIT, Cambridge, USA.

[9] Franses, P.H., 1994. A method to select between Gompertz and Logistic trend curves. Technological Forecasting \& Social Change 46, 45-49.

[10] Gurung, B., Singh, K.N., Shekhawat, R.S., Yeasin, M., 2018. An insight into technology diffusion of tractor through Weibull growth model. Journal of Applied Statistics 45, 682-696.

[11] Hyndman, R.J., Koehler, A.B., 2006. Another look at measures of forecast accuracy. International Journal of Forecasting 22, 679-688.

[12] Kühleitner, M., Brunner, N., Nowak, W.G., Renner-Martin, K., Scheicher, K., 2019. Best fitting tumor growth models of the von Bertalanffy-Pütter Type. BMC Cancer 19, published online: DOI /10.1186/s12885-0195911-y.

[13] Mar-Molinero, C., 1980. Tractors in Spain: a logistic analysis. Journal of the Operational Research Society 31, 141-152.

[14] Marusic, M., Bajzer, Z., 1993. Generalized two-parameter equations of growth. Journal of Mathematical Analysis and Applications 179, 446-462.

[15] Meade, N., 1984. The use of growth curves in forecasting market development-a review and appraisal. Journal of Forecasting 3, 429-451.

[16] Monod, J., 1949. The growth of bacterial cultures. Annual Reviews of Microbiology 8, 371-374.

[17] Motulsky, H., Christopoulos, A., 2003. Fitting Models to Biological Data Using Linear and Nonlinear Regression: A Practical Guide to Curve Fitting. Oxford University Press, Oxford, U.K.

[18] Murphy, H., Jaafari, H., Dobrovolny, H.M., 2016. Differences in predictions of ODE models of tumor growth: a cautionary example. BMC Cancer 16, 163-172.

[19] Naseri, M.B., Elliott, G., 2013. The diffusion of online shopping in Australia: Comparing the Bass, Logistic and Gompertz growth models. Journal of Marketing Analytics 1, 49-60. DOI 10.1057/jma.2013.2.

[20] Nguimkeu, P., 2014. A simple selection test between the Gompertz and Logistic growth models. Technological Forecasting \& Social Change 88, 98-105.

[21] Ohnishi, S., Yamakawa, T., Akamine. T., 2014. On the analytical solution for the Pütter-Bertalanffy growth equation. Journal of Theoretical Biology 343, 174-177.

[22] Pell, B., Kuanga, Y., Viboud, C., Chowell, G., 2018. Using phenomenological models for forecasting the 2015 Ebola challenge. Epidemics 22, 62-70.

[23] Pütter, A., 1920. Studien über physiologische Ähnlichkeit. VI. Wachstumsähnlichkeiten. Pflügers Archiv für die Gesamte Physiologie des Menschen und der Tiere 180, 298-340.

[24] Renner-Martin, K., Brunner, N., Kühleitner, M., Nowak, W.G., Scheicher, K., 2018. Optimal and near-optimal exponent-pairs for the Bertalanffy-Pütter growth model. PeerJ 6, published online: DOI 10.7717/peerj.5973. 
[25] Richards, F.J., 1959. A Flexible Growth Function for Empirical Use, Journal of Experimental Botany, 10, 290300.

[26] Satoh, D., Matsumura, R., 2018. Monotonic decrease of upper limit estimated with Gompertz model for data described using logistic model. Japan Journal of Industrial and Applied Mathematics, published online: DOI 10.1007/s13160-018-0333-9.

[27] Solow, R., 1957. Technical Change and the Aggregate Production Function. The Review of Economics and Statistics 39, 312-320.

[28] Táboas, D.L., Fernández-Prieto, L., Geada, A.D., 2019. Agriculture and Agricultural Policies in Spain (19391959). In: Rural History Conference (in preparation). Published online: DOI 10.13140/2.1.1521.3762.

[29] Vidal, R.V.V., 1993. Applied simulated annealing. In: Lecture notes in economics and mathematical systems. Berlin: Springer-Verlag.

[30] West, G.B., Brown, J.H., Enquist, B.J., 2001. A general model for ontogenetic growth. Nature 413, 628-631.

[31] World Bank, 2019. World Bank Open Data, Link: data.worldbank.org (last visit: 01.07.2019)

[32] Yamakawa, P., Rees, G.H., Salas, J.M., Alva, N., 2013. The diffusion of mobile telephones: an empirical analysis for Peru. Telecommunication Policy, 37, 594-606. 